\title{
Longitudinal changes in symptom-based female and male LUTS clusters
}

Cindy L. Amundsen MD ${ }^{1}$ | Margaret E. Helmuth $\mathbf{M A}^{2} \mid$ Abigail R. Smith $\mathbf{P h D}^{2} \mid$ John O.L. DeLancey MD ${ }^{3}$ | Catherine S. Bradley MD, MSCE ${ }^{4}$ Kathryn E. Flynn PhD ${ }^{5}$ | Kimberly S. Kenton MD, MS ${ }^{6}$ | H. Henry Lai MD ${ }^{7}$ (1) | David Cella PhD $^{6}$ | James W. Griffith PhD $^{6}$ | Victor P. Andreev PhD, DSc ${ }^{2} \mid$ J. Eric Jelovsek MD, MMEd ${ }^{1}$ | Alice B. Liu MPH ${ }^{8} \mid$ Ziya Kirkali MD $^{9}$ | Claire C. Yang MD $^{8}{ }^{8}$ | the LURN Study Group

${ }^{1}$ Department of Obstetrics and Gynecology, Duke University Medical Center, Durham, North Carolina

${ }^{2}$ Arbor Research Collaborative for Health, Ann Arbor, Michigan

${ }^{3}$ University of Michigan, Ann Arbor, Michigan

${ }^{4}$ University of Iowa Carver College of Medicine, Iowa City, Iowa

${ }^{5}$ Medical College of Wisconsin,

Milwaukee, Wisconsin

${ }^{6}$ Northwestern University Feinberg School of Medicine, Chicago, Illinois

${ }^{7}$ Washington University School of Medicine, St. Louis, Missouri

${ }^{8}$ University of Washington, Seattle, Washington

${ }^{9}$ National Institute of Diabetes and Digestive and Kidney Diseases, Bethesda, Maryland

\section{Correspondence}

Cindy L. Amundsen, MD, Department of Obstetrics and Gynecology, Duke University Medical Center, 5324 McFarland Drive, Suite 310, Durham 27707, NC.

Email: cindy.amundsen@duke.edu

\section{Funding information}

National Institutes of Health's National Center for Advancing Translational Sciences; National Institute of Diabetes and Digestive and Kidney Diseases, Grant/Award Numbers: DK097772, DK097776, DK097779, DK097780, DK099879, DK099932, DK100011, DK100017

\begin{abstract}
Aims: Lower urinary tract symptoms (LUTS) are diverse in type and variable in severity. We examined symptom change within the Symptoms of the Lower Urinary Tract Dysfunction Research Network (LURN) Observational Cohort study identified clusters over time and tested associations with treatments received.
\end{abstract}

Methods: Patient-reported LUTS and treatment data were collected at multiple time points between baseline and 12 months from the LURN Observational Cohort study. LUTS severity scores were calculated to summarize changes in symptom reporting over time in previously identified LURN clusters. Repeated measures linear regression models tested adjusted associations between cluster membership and severity scores.

Results: Four-hundred seventeen men and 396 women were classified into improved, unchanged, and worsened symptoms between baseline and 12 months (men: 44.1\%, 40.5\%, and 15.3\%; women: 55.8\%, 33.1\%, $11.1 \%$, respectively). Improvement in LUTS severity scores varied by cluster (estimated adjusted mean change from baseline range: -.04 change in standard deviations of severity scores $(\Delta \mathrm{SD})$ to $-.67 \Delta \mathrm{SD})$. Prostate surgery was associated with improved severity scores $(-.63 \Delta \mathrm{SD})$ in men, while stress incontinence surgery was associated with improved severity scores $(-.88 \Delta \mathrm{SD})$ in women.

Conclusion: Symptom improvement varied by cluster indicating response to therapy differs amongst subtypes of patients with LUTS. The differential improvement of patients in clusters suggests mechanistic differences between clusters and may aid in selecting more targeted treatments in the future.

\section{KEY Y O R D S}

lower urinary tract symptoms, symptom-based clustering, the Symptoms of Lower Urinary Tract Dysfunction Research Network Observational Cohort study, urinary incontinence 


\section{1 | INTRODUCTION}

Lower urinary tract symptoms (LUTS) are diverse in quality and variable in severity, however, the current treatment of LUTS is primarily based on the predominant symptom. Treatment decisions are largely a matter of patient preference and alternative treatments are recommended only when improvement is insufficient and symptoms are severe, resulting in a notable failure rate. ${ }^{1}$ In reality, many individuals report multiple urinary symptoms, and it might be that individuals with certain groups of symptoms should be assessed for treatment efficacy, rather than basing treatment efficacy on a single or predominant symptom.

The Symptoms of Lower Urinary Tract Dysfunction Research Network (LURN) was assembled in 2012 to increase our understanding of LUTS by identifying the important subtypes of patients with LUTS. ${ }^{2,3}$ The rationale for defining subtypes was the recognition that patients with LUTS are a heterogeneous group with regard to presentation, severity, and symptom progression over time. LURN's first step to defining patient subtypes was to use a resampling-based consensus clustering approach. This, using the patient baseline reported symptom data, allowed for the identification of novel symptom-based clusters. ${ }^{4}$ Four male and four female clusters were identified, providing the basis for our current analysis. The first male cluster (M1) was the largest of the four male clusters and reported moderate to severe levels of storage and voiding symptoms, while the second male cluster (M2) reported moderate postmicturition symptoms (postvoid dribbling and postvoid leaking) along with some weak stream symptoms. The third male cluster (M3) was the least symptomatic, endorsing mostly moderate frequency symptoms. The fourth male cluster (M4) reported severe frequency, urgency, and urgency incontinence symptoms.

As in males, the four female clusters were also characterized by multiple symptoms. The first female cluster (F1) reported frequency, postmicturition, and voiding symptoms, at a mild to moderate level. The second female cluster (F2) endorsed more severe frequency and urgency, as well as urgency incontinence. The third female cluster (F3), the largest of the four female clusters, reported moderate to severe levels of incontinence, in addition to frequency, urgency and mild voiding symptoms. The fourth female cluster (F4) reported moderate to severe levels of all symptoms (Table S1).

Because complex conditions such as LUTS may be better understood by subtyping patients, LURN's conceptual framework incorporates the investigation of patients with LUTS at multiple levels, including patient's symptoms, characteristics of the genitourinary organs and comorbidities, and cellular/molecular factors whereby meaningful differences among patients may be identified. ${ }^{2}$ The male and female clusters were found to differ on self-reported mental health, bowel function, and sexual function measures, indicating that distinct patient subtypes were identified with the symptom-based clusters. $^{3,4}$ In this analysis we examined the differential changes in symptom severity by a cluster when treated with standard therapy. But since symptoms are just one component to the complexity of lower urinary tract dysfunction, further redefining of these clusters by incorporating additional data will be necessary to understand the mechanisms of each subtype.

The objective of this analysis is to assess how the symptom profiles of patients presenting with similar combinations of LUTS at baseline change over time. We sought to identify symptom change within the LURN identified clusters in the 12 months after the presentation and test associations with treatments received.

\section{MATERIALS AND METHODS}

\subsection{Study design and population}

The LURN Observational Cohort Study is a 1 year, multicenter study. Men and women over 18 years presenting to a LURN clinic with at least one LUTS as reported on the LUTS Tool ${ }^{5}$ were enrolled. Patients were not required to be treatment-naive at enrollment. Patient's medical history, self-reported LUTS, physical exam findings and patient-reported outcome (PRO) questionnaires reporting on bowel functioning, psychological health, urologic pain, pelvic floor, and sexual and erectile function were collected at the baseline visit. Follow-up in clinic visits at 3 and 12 months collected data on treatment history for LUTS and other PROs. Additional phone visits collected treatment history for LUTS at 6 and 9 months. ${ }^{6}$

\section{$2.2 \mid$ Measures}

Self-reported LUTS were recorded using the LUTS Tool and the American Urological Association Symptom Index (AUA-SI). The LUTS Tool is a 44-item questionnaire assessing the severity and bother of 22 urinary symptoms. The questionnaires collected include the PROMIS gastrointestinal constipation, diarrhea, and bowel incontinence subsets, ${ }^{7}$ PROMIS Depression and Anxiety Short Forms, ${ }^{8}$ PROMIS Sleep Disturbance Short Form, ${ }^{9}$ Perceived Stress Scale, ${ }^{10}$ Genitourinary Pain Index ${ }^{11}$ Pelvic Floor Distress Inventory, ${ }^{12}$ International Index of Erectile Function, ${ }^{13}$ and Pelvic Organ Prolapse/Incontinence Sexual Questionnaire, IUGA-revised. ${ }^{14}$ 
LUTS treatments information before enrollment were collected at baseline and patients subsequently reported specific LUTS treatments (surgeries, medications, behavioral or physical therapies) at 3, 6, 9, and 12 months. Specific treatments were collapsed into categories relating to either the type of treatment (eg, nutraceutical/ herbal remedies) or target symptom (eg, medication for overactive bladder; Table S2). Participants were treated by their physicians according to standard care practices.

An overall LUTS Tool severity score used to show the total LUTS severity, was calculated as the Euclidean length of all LUTS Tool severity questions and was weighted by the correlations between baseline LUTS Tool responses, to account for redundancy between questions. ${ }^{15}$ The overall LUTS Tool severity score was scaled from 0 to 100 , with 0 being the least severe (no symptoms), and 100 being the most severe (most severe rating for all 22 symptoms). In addition, a cluster-specific severity score was calculated using the same method separately for each cluster only including those symptoms highly endorsed by that cluster at baseline. "High endorsement" was defined as a median cluster response of "sometimes" or greater (Table S3). This score was used to show the combined severity of symptoms that were highly endorsed within each cluster, which were likely to be the focus of treatment. Using both scales we are able to observe changes in the most severe symptoms for each patient (cluster-specific scale) in addition to capturing changes in new symptoms that may develop over time (overall severity scale).

The cluster-specific severity score was used to classify participants based on changes in the most severe symptoms within each cluster. Participants were classified into improved, unchanged, or worsened symptom severity at 12 months by comparing baseline and 12-month cluster-specific severity scores. A decrease or increase in cluster-specific severity score of more than $1 / 2$ of a standard deviation of the baseline score was defined as improved or worsened, respectively, as this is generally accepted as a "clinically meaningful difference." ${ }^{16}$ Participants with cluster-specific severity scores at 12 months within $1 / 2$ standard deviation of their baseline score were classified as having unchanged symptom severity at baseline and 12 months.

\section{3 | Statistical analysis}

For participants completing at least two-thirds of the LUTS Tool at each visit, multiple imputations were performed on self-reported data due to data missingness between $1 \%$ and $6 \%$ for LUTS Tool questions. Ten imputed datasets were generated using a sequential regression technique in IVEware version $2.0 .^{17,18}$
Participants who met the threshold to have imputation performed at baseline, 3 and 12 months were included in all analyses. Participant characteristics were reported as means and standard deviations or medians and interquartile ranges, as appropriate, for continuous variables, and percentages for categorical variables, stratified by improvement status and sex. Comparisons between improvement status within each sex were made using the $\chi^{2}$ tests and Wilcoxon two-sample tests or KruskalWallis tests for categorical and continuous variables, respectively. Comparisons between baseline and 12month LUTS symptoms, stratified by cluster, were made using Wilcoxon two-sample tests, with an adjustment for multiple comparisons using the false discovery rate correction. ${ }^{19}$

Repeated measures linear regression models were used to assess associations between severity scores measured at baseline, 3 and 12 months, and baseline patient characteristics including age, race, ethnicity, body mass index, comorbidities (as assessed by the functional comorbidity index), alcohol consumption, smoking status, education, and marital status, PRO's including bowel functioning, psychological symptoms and sleep disturbance, and cluster membership. Treatments received over the course of the study period were also included as time-dependent covariates. Repeated measures linear regression models allow for the inclusion of treatments for the same patient at multiple time points, which accounts for patients who received multiple, sequential treatments over the course of the study, resulting in a better estimate of the association between severity score and specific treatments. The model also controls for the nonindependence of multiple time points for each patient.

Separate models were fitted for men and women for the overall severity score. Stepwise selection with entrylevel at $P=.15$ and stay level at $P=.20$ was used for variable selection. Interactions between visit and highly reported treatment ( $>10 \%$ use in each cluster) and visit and cluster membership were tested in all models to assess differences in severity score trajectories over time by treatment use and cluster membership. Parameter estimates for differences in severity scores are reported on the per-unit (raw score) and per-standard deviation of the severity score (ie, effect size) scale. All statistical analyses were performed using SAS version 9.4 (SAS Institute Inc, Cary, NY).

\section{3 | RESULTS}

Among 813 participants with sufficiently completed questionnaires at all three time points that were eligible 


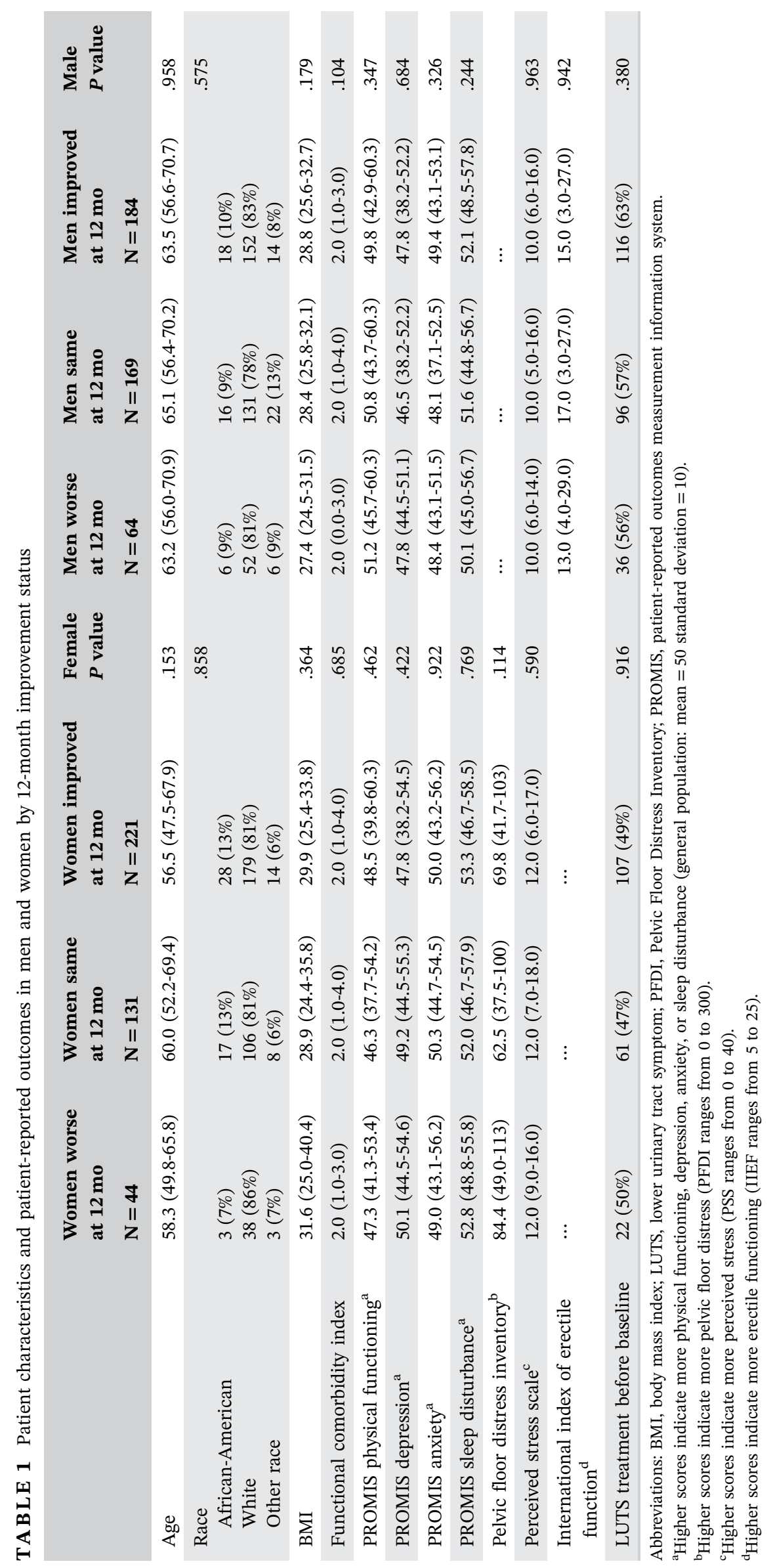


for analysis, 184 (44.1\%), 169 (40.5\%), and 64 (15.3\%) men and 221 (55.8\%), 131 (33.1\%), and 44 (11.1\%) women were classified as improved, unchanged, and worsened, respectively, based on change in cluster-specific severity score from baseline to 12 months. The majority were white, married or living with a partner, overweight or obese, and middle age (Tables 1 and S4). There were no baseline demographic or clinical differences between improved, unchanged, or worsened groups with the exception of ethnicity in women, with Hispanic women more likely to be improved at 12 months; and marital status in men, with men who were married or living with a partner, were more likely to have unchanged symptom severity between baseline and 12 months.

Radar plots, presented in Figure 1, show baseline and 12-month symptom signatures for each of the clusters. At baseline, clusters M1, M4, F3, and F4 presented with many symptoms (range: 10-18) with median severity reported as "sometimes" or greater, while clusters M2, M3, F1, and F2 presented with fewer symptoms (range: 2-6) with median severity reported as "sometimes" or greater. At 12 months, there were statistically significant decreases in postmicturition $(-1.3$ unit change $[\Delta]$ in average LUTS Tool response, $P=.004)$, voiding $(-1.7 \Delta$, $P=.02)$, pain $(-0.8 \Delta, \quad P=.02)$, urgency $(-1.3 \Delta$, $P<.001)$, and frequency symptoms $(-1.4 \Delta, P=.01)$ in cluster $\mathrm{M} 1$, in urgency $(-1.3 \Delta, P<.001)$ and incontinence symptoms $(-1.2 \Delta, P=.02)$ in cluster M4, in postmicturition symptoms $(-0.9 \Delta, P=.05)$ in cluster M2 and in frequency symptoms $(-0.9 \Delta, P=.01)$ in cluster M3. Cluster M3 also saw a statistically significant increase in incontinence with urgency $(0.6 \Delta, P=.02)$, but this cluster reported the lowest level of overall symptoms at both time points. Cluster F1 reported significant decreases in postmicturition $(-1.0 \Delta$, $P=.01)$, voiding $(-0.6 \Delta, \quad P=.04)$, pain $(-0.8 \Delta$, $P=.01)$, and frequency symptoms $(-1.1 \Delta, P=0.01)$. Cluster F2 and F3 reported significant decreases in frequency $(-1.5 \Delta, P=.003,-1.3 \Delta, P<.001$, respectively) and urgency symptoms $(-1.4 \Delta, P=.01,-1.5 \Delta$, $P<.001$, respectively), with cluster $\mathrm{F} 3$ also reporting decreases in postmicturition $(-0.7 \Delta, P=.03)$, voiding $(-0.6 \Delta, P=.01)$, and incontinence $(-2.4 \Delta, P=.001)$ symptoms. Cluster F4 saw a significant decrease in frequency $(-1.3 \Delta, P=.01)$, postmicturition $(-1.7 \Delta$, $P=.002)$ and incontinence symptoms $(-1.6 \Delta, P=.04)$.

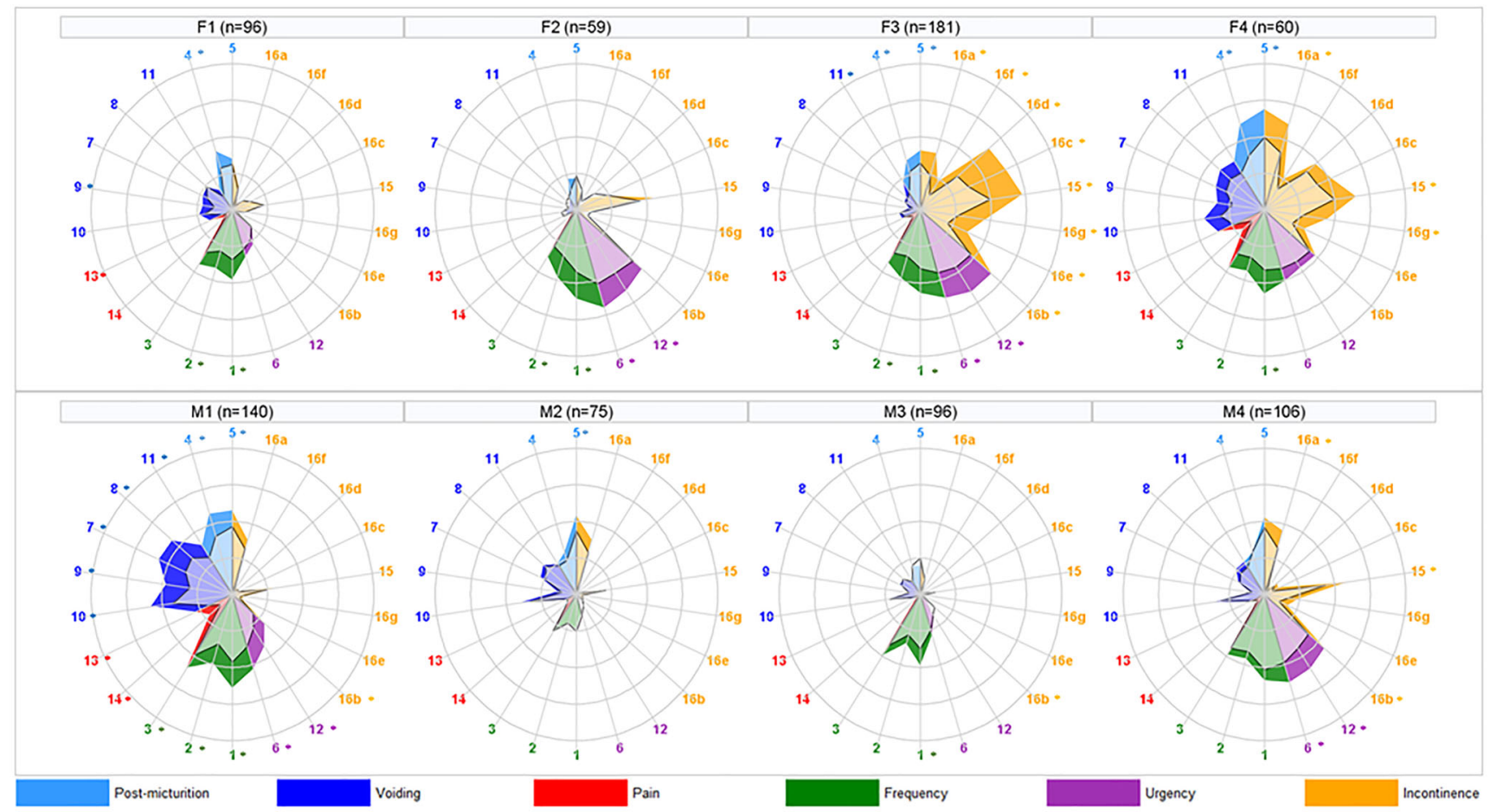

F I G URE 1 Radar plots of mean cluster symptoms reported at baseline and 12 months The exterior colored point on each spoke of the radar plots represent symptoms reported at baseline, while the interior outlined white point on the spoke represents symptoms reported at 12 months. The colored band between these two points represents the change in symptoms between baseline and 12 months. Larger regions of color indicate larger decreases in the mean symptom severity and bother within that cluster. Any symptom where the outlined white section expands beyond the colored section represents symptoms that were reported as on average worse at 12 months compared with baseline. Spokes on the radar plots (symptom items) that were statistically significantly different between baseline and the 12-month visit are denoted by asterisks 
On average, symptoms mostly improved or stayed the same between baseline and 12 months, but there was variability within each symptom category and across clusters.

Comparisons between the overall severity score and the cluster-specific severity scores allowed us to examine the difference between changes in highly reported symptoms within each cluster and change in all LUTS. Changes in cluster-specific severity scores were reflected in the overall severity score as demonstrated by the strong positive relationship between the two scores (Figure S1). This indicates that on average, participants reported stability or slight improvement in their clusterspecific symptoms and minimal changes in other symptoms, and the symptom signatures of the clusters stayed largely the same.

Treatments received differed between men and women. Overall, more men reported medication use (55\% vs $30 \%, P<.001)$, while more women reported behavioral or physical therapy $(74 \%$ vs $30 \%, P<.001)$. More men reported no treatment ( $28 \%$ vs $10 \%$, respectively; $P<.001$ ) (Figure 2). Surgery was less frequently reported in men (8-17\% per cluster) and women (8-31\% per cluster), with the most common procedures being surgery for stress incontinence in F3 (23\%) and prostate surgery in M1 (14\%) (Figures 2 and 3). Women reported a higher proportion of not receiving treatment before enrollment than men (51\% vs $40 \%$, respectively; $P=.002$ ).

Results from multivariable repeated measures linear regression in men demonstrated improvement at 3 and 12 months compared with baseline for all four clusters, but similar scores between 3 and 12 months, indicating that scores tended to level off after initial improvement. The degree of decrease varied by cluster (range: .07-.63 SD decrease), with the largest decreases observed in cluster $\mathrm{M} 1$ at $3(.55 \mathrm{SD})$ and 12 months (.63 SD). Prostate surgery was associated with decreased severity scores (.63 SD) in all clusters. Baseline patient variables associated with worse overall severity scores in men included higher anxiety levels and more sleep disturbance (Table 2).

In women, overall severity scores at 3 and 12 months were lower than baseline for all clusters with a decrease in scores ranging from .20 to .67 SDs. Clusters F3 and F4 had on average adjusted overall severity scores greater than $.5 \mathrm{SD}$ lower than baseline at 12 months. Severity

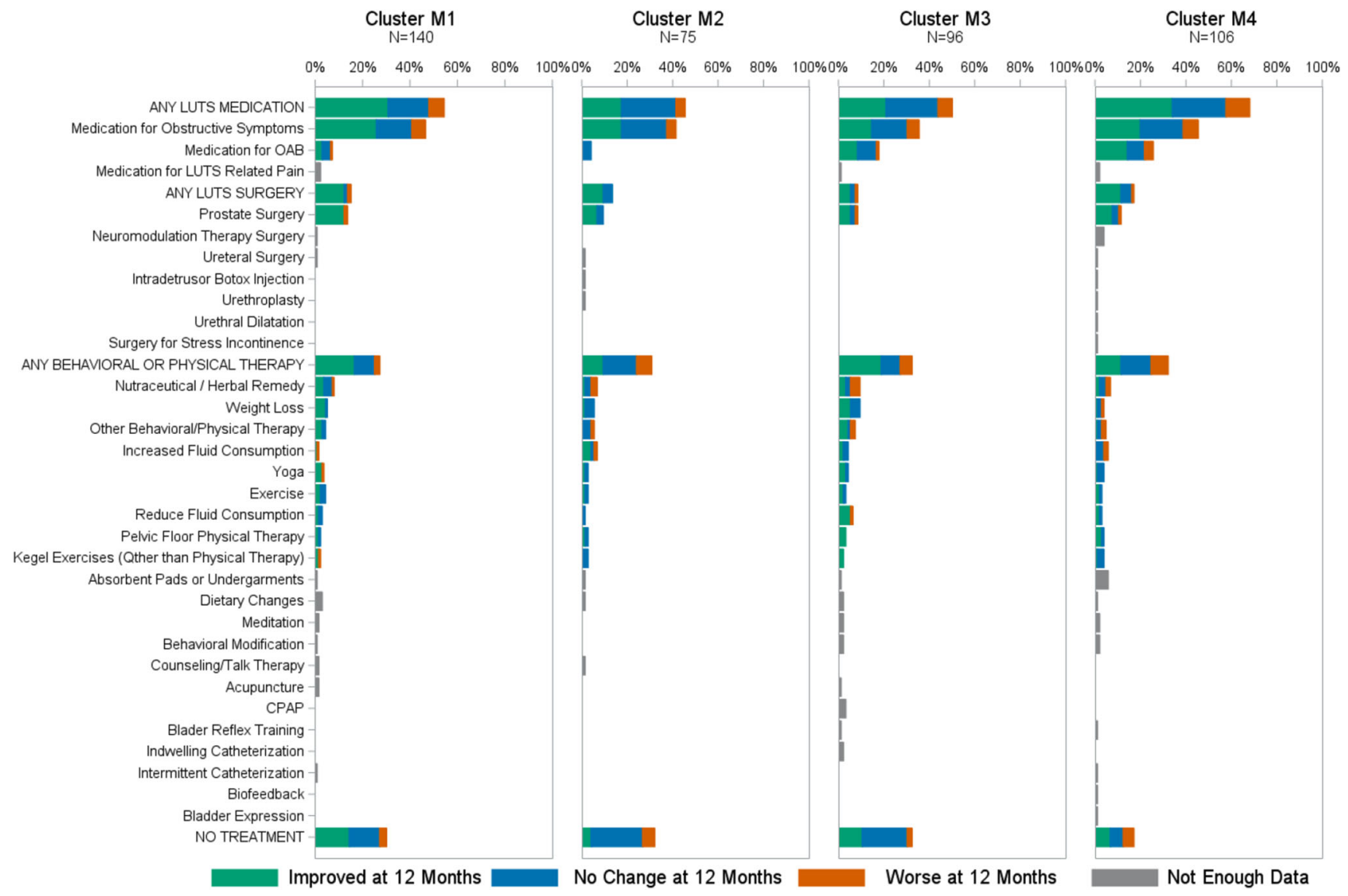

F I G U RE 2 Distribution of treatments across clusters and proportion of patients with changes in symptoms within those treatments: men footnote: overall $28 \%$ reported using more than one treatment ( $17 \%$ reported two treatments, $7 \%$ reported three treatments) 


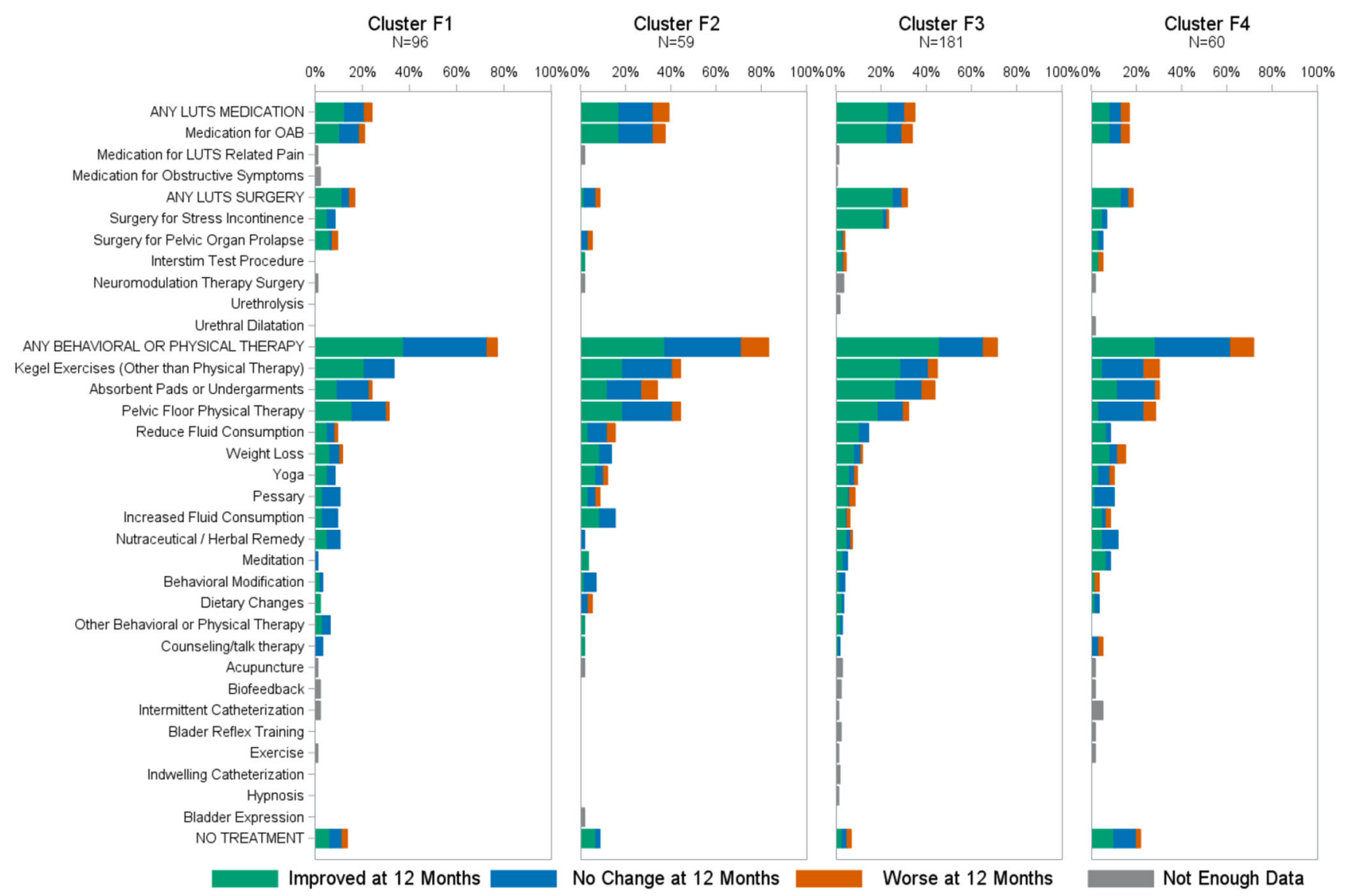

F I G U RE 3 Distribution of treatments across clusters and proportion of patients with changes in symptoms within those treatments: women footnote: overall $62 \%$ reported using more than one treatment (23\% used two treatments, $15 \%$ used three treatments)

score trajectories were similar to those seen in men, with no differences between 3 and 12 months scores detected. Having surgery for stress incontinence was associated with better overall severity scores (.88 SD decrease) in all clusters. In addition, more comorbidities, more sleep disturbance, and worse pelvic floor distress inventory scores (PFDI) (all assessed at baseline) were associated with higher levels of overall severity at all-time points.

\section{DISCUSSION}

Fifty percent of participants in this study showed LUTS improvement during the 1-year follow-up. This low rate of improvement substantiates the need to find a better way to diagnose and treat LUTS and to continue to add additional data to redefine the clusters. Most symptoms showed improvement across all clusters; however, the degree of improvement varied by the cluster. Both the overall LUTS severity score and cluster-specific severity score changed consistently over time and we did not see a substantial increase in severity or bother of a specific LUTS that was not highly endorsed at baseline, which indicates that our cluster signatures remained stable over time. This suggests that the clusters may be mechanistically distinct rather than different stages of LUTS. The incorporation of more diagnostic data to redefine the clusters may help prove this finding.

Despite recruiting from academic urologic and urogynecologic centers, $46 \%$ of the cohorts reported no prior LUTS treatment at baseline; therefore, men and women utilized a wide variety of behavioral treatments in each cluster during the 12-month follow-up. Furthermore, in three of the four male clusters, over $30 \%$ did not report using LUTS therapy during the follow-up period. Interestingly, within the M2 and M4 clusters, the proportions reporting unchanged or worsened symptoms at 12 months were similar for those who did and did not report treatment of any kind. This may be due to low baseline symptoms in those clusters, making a salutary change in symptoms more difficult to detect. Although those with high severity scores at baseline improved the most, there were still a significant number of unchanged or worsened in each cluster, which supports the need to better understand the pathophysiology of these individuals. By continuing to redefine the current LURN 
TABLE 2 Multivariable repeated measures linear regression results

\begin{tabular}{|c|c|c|c|c|c|}
\hline \multirow{2}{*}{ Overall severity score: men } & \multirow[t]{2}{*}{$\begin{array}{l}\text { Mean } \\
\text { estimate }\end{array}$} & \multicolumn{2}{|c|}{$\begin{array}{l}\text { Mean } \\
\text { Confidence limits }\end{array}$} & \multirow[t]{2}{*}{$\begin{array}{l}\text { Standardized } \\
\text { estimate }^{\mathrm{a}}\end{array}$} & \multirow[t]{2}{*}{$P$ value } \\
\hline & & & & & \\
\hline $\begin{array}{l}\text { PROMIS anxiety (per } 5 \text { unit increase) } \\
\text { (centered at a mean score of } 50 \text { ) }\end{array}$ & 1.469 & 0.754 & 2.183 & 0.108 & $<.001$ \\
\hline 3 mo vs baseline: cluster M1 & -7.461 & -9.533 & -5.390 & -0.551 & $<.001$ \\
\hline 3 mo vs baseline: cluster M2 & -2.390 & -4.544 & -0.237 & -0.176 & .030 \\
\hline 3 mo vs baseline: cluster M3 & -0.889 & -2.631 & 0.853 & -0.066 & .317 \\
\hline 3 mo vs baseline: cluster M4 & -3.470 & -5.599 & -1.341 & -0.256 & .001 \\
\hline 12 mo vs baseline: cluster M4 & -4.287 & -6.633 & -1.942 & -0.317 & $<.001$ \\
\hline $\begin{array}{l}\text { Prostate surgery } \\
\text { Overall severity score: women }\end{array}$ & -8.536 & -12.730 & -4.341 & -0.630 & $<.001$ \\
\hline Intercept & 16.980 & 10.968 & 22.992 & $\cdots$ & $<.001$ \\
\hline $\begin{array}{l}\text { Functional comorbidity index (per } 1 \text { unit increase) } \\
\text { (centered at a mean FCI of } 2.4 \text { ) }\end{array}$ & 0.817 & 0.324 & 1.309 & 0.059 & .001 \\
\hline $\begin{array}{l}\text { PROMIS sleep disturbance (per } 5 \text { unit increase) } \\
\text { (centered at a mean score of } 50 \text { ) }\end{array}$ & 0.947 & 0.369 & 1.524 & 0.068 & .001 \\
\hline $\begin{array}{l}\text { PFDI (per } 5 \text { unit increase) (centered at a mean } \\
\text { PFDI score of 77.9) }\end{array}$ & 0.583 & 0.477 & 0.689 & 0.042 & $<.001$ \\
\hline 12 mo vs baseline: cluster F2 & -2.739 & -5.571 & 0.093 & -0.196 & .058 \\
\hline 12 mo vs baseline: cluster F3 & -7.582 & -9.665 & -5.499 & -0.543 & $<.001$ \\
\hline 12 mo vs baseline: cluster F4 & -9.327 & -14.596 & -4.058 & -0.668 & $<.001$ \\
\hline SUI surgery & -12.289 & -16.096 & -8.483 & -0.880 & $<.001$ \\
\hline
\end{tabular}

Note: Bolded covariates highlight clinically meaningful differences in estimates, based on a standardized estimate of greater than 0.5 or less than -0.5 . Abbreviations: PFDI, Pelvic Floor Distress Inventory; PROMIS, patient-reported outcomes measurement information system; SUI, stress urinary incontinence. ${ }^{a}$ Estimates are standardized by the standard deviation of the outcome and represent the mean estimate's proportion of one standard deviation.

clusters by incorporating more data that include diagnostic information and possibly cellular/molecular factors, more meaningful differences among patients may be identified.

Associations between treatments (or combinations of treatments) and symptom changes did not vary by the cluster. We saw an improvement in symptoms for women who had stress incontinence surgery during the study period, regardless of the cluster. However, treatment decisions were made per standard of care without knowledge of cluster membership, therefore further assessment of treatment targeted to cluster membership is needed to determine potential differences in disease etiology and treatment response.

We found that certain factors were predictive of worse symptoms (such as multiple comorbidities, high anxiety, high scores on disease-specific quality of life PROs, and sleep disturbance); nonetheless, we do not understand the underlying pathophysiologies for why these patients might be more difficult to treat and re-emphasizes a need for pathophysiologic studies to better understand LUTS. The largest decreases in overall severity scores 
were in the clusters with more symptoms. For men, prostate surgery was associated with symptom improvement in all clusters; and only for the female cluster (cluster group F3) with a high proportion reporting stress urinary incontinence, did we find midurethral slings correlated with reduced LUTS. Therefore, since various pathophysiologies can produce similar LUTS, we need to understand the underlying causes for an individual to better guide treatment decisions and to find better therapies.

A primary limitation of our study is that our symptom-based clusters need to be refined using additional data (eg, bladder diary and urodynamics data). Work is currently underway to integrate other data domains. In addition, the applicability of these clusters to other populations (eg, noncare seeking) with higher levels of racial and LUTS severity diversity is yet to be determined. Clinical validation of these clusters should be the subject of future studies. Finally, from this observational study design, causal relationships between treatment and symptom change cannot be determined nor can a comparison be made between the different therapies used for similar symptoms. There was variability in the frequency of individual treatments used (eg, midurethral slings as compared with bulking agents) and these results were combined. This does not allow for the assessment of associations between the different individual treatments and symptom severity, which may differ from the group estimates presented. Further prospective studies will be required to evaluate how specific individuals change within each cluster.

\section{5 | CONCLUSIONS}

Symptom improvement varied by cluster indicating response to therapy differs amongst subtypes of patients with LUTS. While specific treatments could not be associated with cluster-specific symptom improvement in this observational study, the differential improvement by cluster can be used to generate further hypotheses regarding mechanistic differences between clusters that could be used to apply more targeted treatments in the future.

\section{ACKNOWLEDGMENTS}

This is publication number 18 of the Symptoms of Lower Urinary Tract Dysfunction Research Network (LURN). The following individuals were instrumental in the planning and conduct of this study at each of the participating institutions.
Duke University, Durham, North Carolina (DK097780): PI: Cindy L. Amundsen, MD, Kevin Weinfurt, PhD; Co-Is: Kathryn Flynn, PhD, Matthew O. Fraser, PhD, Todd Harshbarger, PhD, Eric Jelovsek, MD, Aaron Lentz, MD, Drew Peterson, MD, Nazema Siddiqui, MD, Alison Weidner, MD; Study Coordinators: Carrie Dombeck, MA, Robin Gilliam, MSW, Akira Hayes, Shantae McLean, MPH

University of Iowa, Iowa City, IA (DK097772): PI: Karl Kreder, MD, MBA, Catherine S Bradley, MD, MSCE, CoIs: Bradley A. Erickson, MD, MS, Susan K. Lutgendorf, $\mathrm{PhD}$, Vince Magnotta, PhD, Michael A. O’Donnell, MD, Vivian Sung, MD; Study Coordinator: Ahmad Alzubaidi

Northwestern University, Chicago, IL (DK097779): PIs: David Cella, Brian Helfand, MD, PhD; Co-Is: James W Griffith, PhD, Kimberly Kenton, MD, MS, Christina Lewicky-Gaupp, MD, Todd Parrish, PhD, Jennie Yufen Chen, PhD, Margaret Mueller, MD; Study Coordinators: Sarah Buono, Maria Corona, Beatriz Menendez, Alexis Siurek, Meera Tavathia, Veronica Venezuela, Azra Muftic, Pooja Talaty, Jasmine Nero. Dr. Helfand, Ms. Talaty, and Ms. Nero are at NorthShore University Health System.

University of Michigan Health System, Ann Arbor, MI (DK099932): PI: J Quentin Clemens, MD, FACS, MSCI; Co-Is: Mitch Berger, MD, PhD, John DeLancey, MD, Dee Fenner, MD, Rick Harris, MD, Steve Harte, PhD, Anne P. Cameron, MD, John Wei, MD; Study Coordinators: Morgen Barroso, Linda Drnek, Greg Mowatt, Julie Tumbarello

University of Washington, Seattle Washington (DK100011): PI: Claire Yang, MD; Co-I: John L. Gore, MD, MS; Study Coordinators: Alice Liu, MPH, Brenda Vicars, RN

Washington University in St. Louis, St. Louis Missouri (DK100017): PI: Gerald L. Andriole, MD, H. Henry Lai; Co-I: Joshua Shimony, MD, PhD; Study Coordinators: Susan Mueller, RN, BSN, Heather Wilson, LPN, Deborah Ksiazek, BS, Aleksandra Klim, RN, MHS, CCRC

National Institute of Diabetes and Digestive and Kidney Diseases, Division of Kidney, Urology, and Hematology, Bethesda, MD: Project Scientist: Ziya Kirkali MD; Project Officer: Christopher Mullins PhD; NIH Personnel: Tamara Bavendam, MD, Robert Star, MD, Jenna Norton

Arbor Research Collaborative for Health, Data Coordinating Center (DK097776 and DK099879): PI: Robert Merion, MD, FACS; Co-Is: Victor Andreev, PhD, DSc, Brenda Gillespie, PhD, Gang Liu, PhD, Abigail Smith, PhD; Project Manager: Melissa Fava, MPA, PMP; Clinical Study Process Manager: Peg Hill-Callahan, BS, LSW; Clinical Monitor: Timothy Buck, BS, CCRP; Research Analysts: Margaret Helmuth, MA, Jon Wiseman, MS; Project Associate: Julieanne Lock, MLitt 


\section{CONFLICT OF INTERESTS}

Dr Bradley and Dr DeLancey report grants from NIH/ NIDDK, during the conduct of the study; Dr Jelovsek reports other from UpToDate outside the submitted work; Dr Yang reports grants from National Institutes of Health, during the conduct of the study; Dr Andreev reports grants from NIDDK, during the conduct of the study; Dr Lai reports grants from National Institutes of Health, during the conduct of the study; other from Medtronic, other from Allergan, personal fees from Aquinox, personal fees from Teva, outside the submitted work; Ms. Helmuth reports grants from NIDDK, during the conduct of the study; Dr Smith reports grants from NIDDK, during the conduct of the study.

\section{ORCID}

H. Henry Lai (D) http://orcid.org/0000-0003-2691-994X Claire C. Yang (1D) http://orcid.org/0000-0002-4750-6258

\section{REFERENCES}

1. Gormley EA, Lightner DJ, Burgio KL, et al. Diagnosis and treatment of overactive bladder (non-neurogenic) in adults: AUA/SUFU Guideline. J Urol. 2012;188(6 suppl): 2455-2463.

2. Yang CC, Weinfurt KP, Merion RM, Kirkali Z; LURN Study Group. Symptoms of lower urinary tract dysfunction research network. J Urol. 2016;196:146-152.

3. Andreev VP, Liu G, Yang CC, et al. Symptom based clustering of women in the LURN observational cohort study. $J$ Urol. 2018;200:1323-1331.

4. Liu G, Andreev VP, Helmuth ME. Symptom Based Clustering of Men in the LURN Observational Cohort Study. J Urol. 2019; 202(6):1230-1239.

5. Coyne KS, Barsdorf AI, Thompson C, et al. Moving towards a comprehensive assessment of lower urinary tract symptoms (LUTS). Neurourol Urodyn. 2012;31:448-454.

6. Cameron AP, Lewicky-Gaupp C, Smith AR, et al. Baseline lower urinary tract symptoms in patients enrolled in LURN: a prospective, observational cohort study. J Urol. 2018;199:1023-1031.

7. Spiegel BM, Hays RD, Bolus R, et al. Development of the NIH patient-reported outcomes measurement information system (PROMIS) gastrointestinal symptom scales. Am J Gastroenterol. 2014;109:1804-1814.

8. Pilkonis PA, Choi SW, Reise SP, Stover AM, Riley WT, Cella D; PROMIS Cooperative Group. Item banks for measuring emotional distress from the patient-reported outcomes measurement information system (PROMIS ${ }^{\circledR}$ ): depression, anxiety, and anger. Assessment. 2011;18:263-283.
9. Yu L, Buysse DJ, Germain A, et al. Development of short forms from the PROMIS ${ }^{\mathrm{TM}}$ sleep disturbance and sleep-related impairment item banks. Behav Sleep Med. 2012;10:6-24.

10. Cohen S, Kamarck T, Mermelstein R. A global measure of perceived stress. J Health Soc Behav. 1983;24(4):385-396.

11. Clemens JQ, Calhoun EA, Litwin MS, et al. Validation of a modified National Institutes of Health chronic prostatitis symptom index to assess genitourinary pain in both men and women. Urology. 2009;74:983-987.

12. Barber MD, Chen Z, Lukacz E, et al. Further validation of the short form versions of the Pelvic Floor Distress Inventory (PFDI) and Pelvic Floor Impact Questionnaire (PFIQ). Neurourol Urodyn. 2011;30:541-546.

13. Rosen RC, Riley A, Wagner G, Osterloh IH, Kirkpatrick J, Mishra A. The International Index of Erectile Function (IIEF): a multidimensional scale for assessment of erectile dysfunction. Urology. 1997;49:822-830.

14. Rogers RG, Rockwood TH, Constantine ML, et al. A new measure of sexual function in women with pelvic floor disorders (PFD): the pelvic organ prolapse/incontinence sexual questionnaire, IUGA-Revised (PISQ-IR). Int Urogynecol J. 2013;24:1091-1103.

15. Helmuth ME, Smith AR, Andreev VP, et al. Use of Euclidean length to measure urinary incontinence severity based on the lower urinary tract symptoms tool. Am J Obstet Gynecol. 2018;218:357-359.

16. Norman GR, Sloan JA, Wyrwich KW. Interpretation of changes in health-related quality of life: the remarkable universality of half a standard deviation. Med Care. 2003;41(5):582-592.

17. Raghunathan TE, Lepkowski JM, Van Hoewyk J, Solenberger P. A multivariate technique for multiply imputing missing values using a sequence of regression models. Surv Methodol. 2001;27(1):85-96.

18. Raghunathan TE, Solenberger PW, Van Hoewyk J. IVEware: imputation and variance estimation software. Ann Arbor, MI: Survey Methodology Program, Survey Research Center, Institute for Social Research. University of Michigan; 2002.

19. Benjamini Y, Hochberg Y. Controlling the false discovery rate: a practical and powerful approach to multiple testing. $J$ R Stat Soc Ser B. 1995;57(1):289-300.

\section{SUPPORTING INFORMATION}

Additional supporting information may be found online in the Supporting Information section.

How to cite this article: Amundsen CL, Helmuth ME, Smith AR, et al. Longitudinal changes in symptom-based female and male LUTS clusters. Neurourology and Urodynamics. 2020;39:393-402. https://doi.org/10.1002/nau.24219 